\title{
Paradoxical Actions of the Serotonin Precursor 5-hydroxytryptophan on the Activity of Identified Serotonergic Neurons in a Simple Motor Circuit
}

\author{
David J. Fickbohm and Paul S. Katz \\ Department of Biology, Georgia State University, Atlanta, Georgia 30302
}

Neurotransmitter synthesis is regulated by a variety of factors, yet the effect of altering transmitter content on the operation of neuronal circuits has been relatively unexplored. We used electrophysiological, electrochemical, and immunohistochemical techniques to investigate the effects of augmenting the serotonin (5-HT) content of identified serotonergic neurons embedded in a simple motor circuit. The dorsal swim interneurons (DSIs) are serotonergic neurons intrinsic to the central pattern generator (CPG) for swimming in the mollusc Tritonia diomedea. As expected, treatment with the serotonin precursor 5-hydroxytryptophan (5-HTP) increased the intensity of serotonin immunolabeling and enhanced the potency of synaptic and modulatory actions elicited by the DSIs. It also greatly enhanced the ability of the DSIs to evoke rhythmic CPG activity. After 5-HTP treatment, microvoltammetric measurements indi-

5-HTP, the immediate precursor of serotonin (5-hydroxytryptamine, 5-HT), has clinical significance for various mental disorders (Byerley et al., 1987; Silverstone, 1992; Launay et al., 1994; Handley, 1995). It is available as a nutritional supplement in the United States and has been touted in the popular press as a means of boosting the activity of the serotonin system. At the cellular level, precursor treatment is a well established method for increasing neurotransmitter synthesis (Cottrell and Powell, 1971; McCaman et al., 1984; Audesirk, 1985; Lookingland et al., 1986; Kabotyanskii and Sakharov, 1991). Furthermore, artificially raising neurotransmitter levels can potentiate synaptic release from neurons (Poulain et al., 1986; Pothos et al., 1996). However, the consequences of altering transmitter levels, particularly 5-HT levels, on neuronal activity within neural circuits are poorly understood. To examine the effects of 5-HTP on serotonergic neurons and on their role in circuit operation, we have used a simple neuronal circuit containing identified serotonergic neurons.

The system that we used is the small, well characterized central pattern generator (CPG) circuit for escape swimming in the nudibranch mollusc Tritonia diomedea (Fig. 1A) (Willows and Hoyle, 1969; Getting, 1989a). The output of the interneurons

\footnotetext{
Received Sept. 15, 1999; revised Dec. 6, 1999; accepted Dec. 7, 1999.

This work was supported by National Institutes of Health Grant NS35371. We thank E. A. Kabotyanski for suggesting the use of 5-HTP and W. N. Frost for many helpful discussions. We thank S. L. Pallas, R. M. Harris-Warrick, D. H. Edwards, and S. Clemens for critiquing earlier versions of this manuscript and Ye Yang and Minhao Zhou for assistance with the immunohistochemistry. We thank A. O. Dennis Willows and Friday Harbor Laboratories for help and support.

Correspondence should be addressed to David Fickbohm, 402 Kell Hall, 24 Peachtree Center Avenue, Atlanta, GA 30303. E-mail: biodjf@panther.gsu.edu. Copyright (C) 2000 Society for Neuroscience $0270-6474 / 00 / 201622-13 \$ 15.00 / 0$
}

cated an increase in a putative 5-HT electrochemical signal during swim CPG activation. Paradoxically, the spiking activity of the serotonergic neurons decreased to a single burst at the onset of the rhythmic motor program, whereas the overall duration of the episode remained about the same. 5-HTP treatment gradually reduced the rhythmicity of the CPG output. Thus, more serotonin did not result in a more robust swim motor program, suggesting that serotonin synthesis must be kept within certain limits for the circuit to function correctly and indicating that altering neurotransmitter synthesis can have serious consequences for the output of neural networks.

Key words: intrinsic neuromodulation; central pattern generator; neurotransmitter content; serotonin immunoreactivity; Tritonia diomedea; synaptic actions; microvoltammetry; confocal fluorescence microscopy

comprising the CPG (Fig. $1 B$ ) directly reflects the motor neuron activity underlying the rhythmic body flexions of the swim (Dorsett et al., 1969; Abraham and Willows; 1971). Generation of the escape swim motor program is dependent on 5-HT release (McClellan et al., 1994). Many cells in the ganglia comprising the brain of Tritonia contain 5-HT (Fig. 1C) (McCaman et al., 1984; McClellan et al., 1994; Sudlow et al., 1998) and are involved in controlling a variety of behaviors. One set of physiologically identified serotonergic neurons, the dorsal swim interneurons (DSIs), is intrinsic to the swim CPG (Getting, 1977, 1989a,b; McClellan et al., 1994; Katz et al., 1994). The DSIs fire repetitive bursts of action potentials (APs) during the swim motor program, contributing to the dorsal flexion phase of the behavior (Fig. 1B) (Getting et al., 1980; Getting, 1981; Getting and Dekin, 1985).

5-HT released from the DSIs plays a dual role. It serves as a conventional neurotransmitter, evoking fast and slow synaptic potentials (Katz and Frost, 1995b). 5-HT also acts a neuromodulator (Katz, 1999), increasing the cellular excitability and synaptic strength of other CPG neurons (Katz et al., 1994; Katz and Frost, 1995a,b, 1997).

In this circuit, individual serotonergic neurons and their actions on postsynaptic targets are stable and can be monitored for days, allowing assessment of changes in serotonergic synaptic strength after precursor treatment. In this regard, the Tritonia swim CPG is a model system for studying 5-HT supplementation effects on neuronal circuit output. The results of such studies may have implications for more complicated vertebrate systems. Our findings indicate that although increasing the level of serotonin dramatically enhances the potency of serotonergic neurons, feedback from other circuit elements results in a decrease in their 

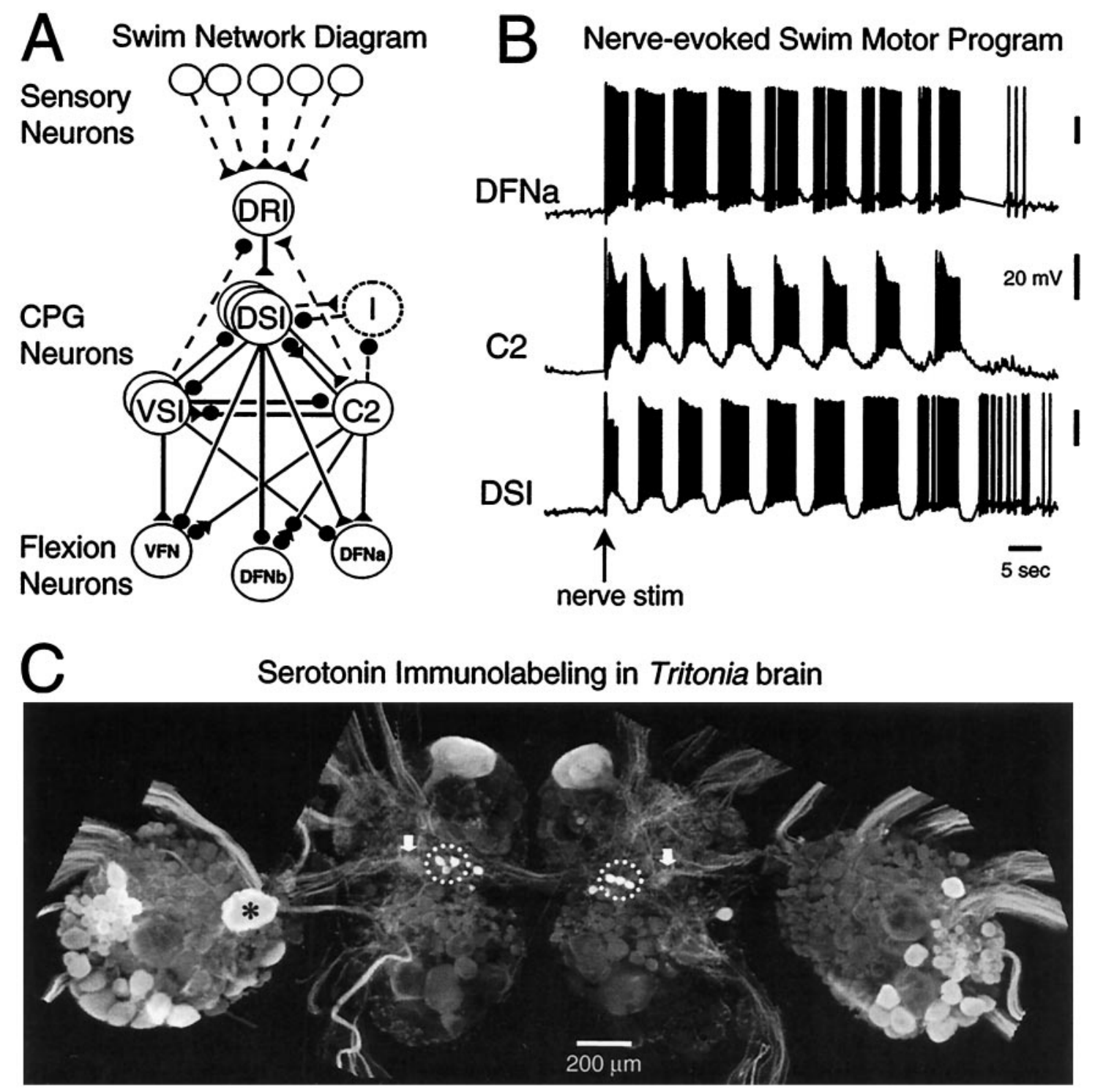

Figure 1. The neurons comprising the swim network of Tritonia mediate production of the rhythmic escape swim pattern. $A$, Schematic of the swim CPG with input and output connections. Sensory input is relayed through the dorsal ramp interneuron (DRI) to the CPG, which consists of the dorsal and ventral swim interneurons (DSIs and VSIs) and cerebral interneuron 2 (C2). The swim interneurons influence the activity of swim flexion neurons: the ventral flexion neurons (VFN) and dorsal flexion neurons ( $D F N a$ and $D F N b)$. Synapses are represented as excitatory (triangles), inhibitory (circles), or multicomponent (triangles and circles). Polysynaptic or unidentified cells and connections are represented as dotted lines. The DSIs recruit inhibition from unidentified inhibitory neurons (I, dotted circle). Each neuron shown in this diagram has a contralateral homolog. B, Simultaneous intracellular recordings from a DF Na, C2, and DSI display the nerve-evoked escape swim motor program in an untreated isolated nervous system. At the arrow, pedal nerve 3 was stimulated with $2 \mathrm{msec}$ voltage pulses at $10 \mathrm{~Hz}$ for $1 \mathrm{sec}$. $C$, Confocal image of serotonin immunoreactivity in the brain of Tritonia. Shown are the left and right fused cerebropleural ganglia (central portion of figure) and pedal ganglia (lateral). The DSIs are situated in the cerebropleural ganglia (dotted circles). Note that not all 5-HT-immunoreactive cells are present bilaterally (e.g., the large cell marked by an asterisk in left pedal ganglion). The putative areas of 5-HT release from the DSIs within serotonergic neuropil areas are indicated by the arrows.

participation in CPG activity and ultimately in a disruption of the motor pattern.

\section{MATERIALS AND METHODS}

Preparation. Specimens of Tritonia diomedea were obtained from Living Elements Limited (Vancouver, British Columbia, Canada). The CNS, consisting of left and right fused cerebropleural and pedal ganglia and associated nerve roots, was removed from animals and immediately placed in normal saline chilled to $2^{\circ} \mathrm{C}$. Normal saline consisted of (in mM:) $420 \mathrm{NaCl}, 10 \mathrm{KCl}, 10 \mathrm{CaCl}_{2}, 50 \mathrm{MgCl}_{2}, 10 \mathrm{D}$-glucose, and 10 HEPES, pH 7.6. The ganglia were pinned down in a Sylgard-lined chamber. After clearing off connective tissue, removing the ganglionic sheath, and attaching suction electrodes to nerves, the preparation was returned to its physiological temperature of $10^{\circ} \mathrm{C}$ through continuous superfusion with chilled saline. Isolated ganglia were allowed to sit unstimulated overnight before use in electrophysiology experiments. For experiments examining synaptic potentials, high divalent cation saline consisting of (in mM:) $285 \mathrm{NaCl}, 10 \mathrm{KCl}, 25 \mathrm{CaCl}_{2}, 125 \mathrm{MgCl}_{2}, 10$ D-glucose, and 10 HEPES, $\mathrm{pH}$ 7.6, was used to increase firing thresholds and thus decrease the contribution of polysynaptic connections. The drugs, 5-HTP (L-2-amino-3[5-hydroxyindolyl]-propionic acid) and 5-HT (serotonin creatinine sulfate) (Sigma, St. Louis, MO) were applied to the bath by switching superfusion inflow reservoirs. The superfusion flow rate was $1-1.5 \mathrm{ml} / \mathrm{min}$. The estimated time for complete bath exchange was 4 min. In most experiments, 5-HTP was applied at 1 or $2 \mathrm{~mm}$ for 30-60 min. However, similar effects are seen after treatment with a lower concentration of 5-HTP (10 $\mu \mathrm{M}, n=3)$ (S. Clemens, personal communication). 
Electrophysiology. Neurons were impaled with glass microelectrodes filled with $4 \mathrm{M}$ potassium acetate and having resistances of 5-15 M $\Omega$. Up to four simultaneous intracellular recordings were made using a pair of AxoClamp 2B (Axon Instruments, Foster City, CA) amplifiers. Recordings were digitized at $>1 \mathrm{kHz}$ and stored on a Pentium computer using hardware and software from Cambridge Electronic Design Limited.

Neurons were identified using physical and electrophysiological criteria, including location, coloration, synaptic connectivity, and participation in fictive swims (Getting, 1977; Taghert and Willows, 1978; Getting et al., 1980). We typically recorded from the same individual identified neurons on two or three consecutive days to monitor the effects of experimental manipulations. A preparation was considered to be in the "rested" state if it had not produced a swim motor program in the previous $3 \mathrm{hr}$.

Swim CPG activity corresponding to a fictive swim was elicited with electrical stimulation (10-15 pulses, $2 \mathrm{msec}$ pulse duration, 5-10 V, $10-20 \mathrm{~Hz}$ ) of left or right pedal nerve 3 (PdN3) through a suction electrode. The swim motor program was defined as the nerve-evoked swim CPG activity beginning at the nerve stimulus and ending at the last major hyperpolarization of DSI (corresponding to the last ventral phase).

APs were elicited at defined frequencies (as indicated) in DSIs or cerebral interneuron $2(\mathrm{C} 2)$ by intracellular current injection with very brief pulses (7-10 nA, 20 msec duration) that each triggered a single AP. EPSPs evoked by stimulation (five pulses at $5 \mathrm{~Hz}$, every $60 \mathrm{sec}$ ) of DSI or $\mathrm{C} 2$ in synaptic followers were measured in high divalent cation saline to limit activation of polysynaptic pathways. The amplitudes of summated EPSPs (i.e., compound EPSPs evoked by a DSI AP train) were measured as the voltage excursion from baseline to peak. The amplitudes of individual EPSPs were measured either from a stable baseline (for initial or single EPSPs) or from a falling baseline (for subsequent EPSPs in a train). Facilitation of EPSP amplitude was measured as the percentage of the third EPSP amplitude compared to the first EPSP amplitude in a train of EPSPs evoked by $5 \mathrm{~Hz}$ DSI activity.

To examine the effect of 5-HTP on the modulatory actions of DSI stimulation while avoiding unwanted activation of polysynaptic pathways and rhythmic bursting in CPG neurons, parameters were chosen to be less intense than those previously used (Katz et al., 1994; Katz and Frost, 1995a,b, 1997). To determine the effect of 5-HTP treatment on DSI modulation of interneuron $\mathrm{C} 2$ excitability in normal saline, $\mathrm{C} 2$ was depolarized via current injection $(+2 \mathrm{nA}, 2 \mathrm{sec}$ duration) every $60 \mathrm{sec}$, and the average instantaneous spike frequency (inverse interspike interval) for C2 was calculated. Every $240 \mathrm{sec}$, the C2 stimulation was preceded by DSI activity ( 15 pulses at $5 \mathrm{~Hz}$ ), ending $2 \mathrm{sec}$ before C2 depolarization. The effect of 5-HTP treatment on the potency of DSI modulation of $\mathrm{C} 2$ synaptic strength was examined in high divalent cation saline. $\mathrm{C} 2$ was stimulated to fire a train of APs (five pulses at $5 \mathrm{~Hz}$ ) at 60 sec intervals. The synaptic strength of $\mathrm{C} 2$ was measured as the summated amplitude of EPSPs (baseline to peak) evoked by $\mathrm{C} 2$ in the dorsal flexion neuron (DFNa). DFNa was held at $-70 \mathrm{mV}$ with two-electrode current clamp. A train of APs $(5-15$ pulses at $5-10 \mathrm{~Hz})$ was stimulated in DSI every $120 \mathrm{sec}$, ending $2-6 \mathrm{sec}$ before $\mathrm{C} 2$ activity. In two of the seven experiments performed for this analysis, gramine (100 uM, Sigma) was used to block the fast synaptic potentials evoked by DSI stimulation. Gramine does not block the neuromodulation by DSI of the synaptic strength of C2 (Katz and Frost, 1995b). The results of these experiments were the same as experiments done without gramine present and were combined for analysis. The amplitude of the summated EPSPs was measured either from a stable baseline or from a falling baseline where appropriate.

For the electrophysiology experiments examining the effects of 5-HTP treatment on nerve-evoked swim CPG activity, DSI synaptic strength, and DSI neuromodulation of C2 excitability and synaptic strength, each preparation served as its own internal control. Thus, the same cells were recorded from before and after 5-HTP treatment.

Electrochemistry. Microvoltammetric measurements of 5-HT-like signals were measured with an IVEC-10 (Harvard Apparatus, Holliston, MA) in chronoamperometry mode (O'Neill, 1994). Single Nafion-coated carbon fiber electrodes ( $8 \mu \mathrm{m}$ diameter, $50 \mu \mathrm{m}$ length) were inserted 50 $\mu \mathrm{m}$ into areas of the cerebropleural ganglion that have previously been determined to contain axonal branches of the DSIs (Getting et al., 1980). Oxidation and reduction currents evoked by $5 \mathrm{~Hz}$ square voltage steps $(0$ $\mathrm{V}$ to $+650 \mathrm{mV}, 50 \%$ duty cycle) were integrated and averaged once per second by IVEC-10 software. Before each experiment, the system was calibrated by measuring the current generated by exposure to varying concentrations of 5-HT (0-2 $\mu \mathrm{M}$, in normal saline). Changes in signal observed after nerve stimulation are relative and do not represent absolute 5-HT concentration. Although the oxidation and reduction currents are typical of 5-HT, and the anion-repelling Nafion coating increases specificity, lacking further characterization, the measured signals may represent the release or production of electroactive compounds in addition to 5-HT. Therefore, we have represented them as arbitrary concentration units (ACU) for which one $\mathrm{ACU}$ is the equivalent of the signal produced by $1 \mu \mathrm{M} 5$-HT in vitro. Furthermore, the signal is almost certainly attributable to overflow from synapses rather than a direct measure of synaptic release, which presumably takes place in a much smaller area and over a faster time scale.

Immunohistochemistry. Immunohistochemistry was performed on tissue treated with $0.5 \%$ type XIV protease for $5 \mathrm{~min}$ and fixed overnight at $4^{\circ} \mathrm{C}$ in paraformaldehyde-lysine-periodate fixative (McLean and $\mathrm{Na}-$ kane, 1974). Ganglia were washed by rinsing two times $(30 \mathrm{~min})$ with cacodylate buffer [0.2 M cacodylic acid (Na salt) in $0.3 \mathrm{~m} \mathrm{NaCl}, \mathrm{pH} 7.5$ ], followed by two rinses ( $30 \mathrm{~min}$ ) with $4 \%$ Triton X-100 in PBS (consisting of $50 \mathrm{mM} \mathrm{Na}_{2} \mathrm{HPO}_{4}$ and $140 \mathrm{mM} \mathrm{NaCl}, \mathrm{pH} 7.2$ ). The ganglia were then incubated in antiserum diluent (ASD; consisting of $0.5 \%$ Triton X-100, $1 \%$ normal goat serum, and $1 \%$ bovine serum in PBS) for $1 \mathrm{hr}$. The preparations were incubated for $72 \mathrm{hr}$ in primary antiserum (rabbit anti-5HT; DiaSorin, Stillwater, MN) diluted in ASD at 1:10,000 for quantification of 5-HT levels or 1:1000 to enhance visualization of neurons with low levels of 5-HT immunoreactivity. After rinsing in $0.5 \%$ Triton X-100 in PBS (6 hr), the ganglia were exposed overnight to secondary antibody (goat anti-rabbit conjugated to Cy2; Jackson ImmunoResearch, West Grove, PA) diluted 1:50 in ASD. After this, the ganglia were washed with $0.5 \%$ Triton X-100 (in PBS) for $6 \mathrm{hr}$, dehydrated in a graded series of ethanol washes, cleared in methyl salicylate, and mounted and coverslipped on microscope slides using Cytoseal 60 (Stephens Scientific, Kalamazoo, MI).

5-HT immunofluorescence was visualized with a laser-scanning confocal microscope (LSM 510; Carl Zeiss, Thornwood, NY) using a 10 or $20 \times$ objective, argon laser excitation at $488 \mathrm{~nm}$, and a $505 \mathrm{~nm}$ long-pass emission filter. Within each processing group, ganglia were dissected from animals within minutes of each other and placed in perfusion chambers, as described above. The experimental ganglia received $2 \mathrm{~mm}$ 5-HTP for $60 \mathrm{~min}$, whereas the control ganglia continued to receive normal saline during that time. Both control and experimental ganglia were washed for $20 \mathrm{hr}$, fixed, and processed for immunohistochemistry in parallel, using aliquots of the same solutions, and visualized using identical confocal microscope settings (gain, scan rate, etc.) to minimize fluorescent signal variability. We measured 5-HT immunofluorescence intensity in cell bodies or groups of cell bodies by averaging the pixel intensity within a defined border from 8-bit $y$-axis maximum projection images using the LSM510 software. Background fluorescence (measured from nonstaining cell bodies) was subtracted from all intensity measurements. The mean intensity of the neurons within a processing group was in arbitrary intensity units $(0-255$ range). These values are strictly comparable only for preparations within a processing group. To compare intensities among processing groups, neuronal intensities in each group were normalized by dividing the average intensity of each cell body by the highest average cellular intensity in the group. The unitless, normalized intensity values are thus in the range of 0.00 (background staining level) to 1.00 (maximum for that group). Note that because of the inherently nonlinear nature of immunohistochemistry, these measurements of immunofluorescence intensity may only be taken as an estimation of 5-HT levels in cells and do not represent actual 5-HT content. However, the technique does allow comparisons of relative 5-HT immunoreactivity, sufficient to determine if precursor treatment alters 5-HT levels.

Statistics. All values given as mean \pm SEM. SigmaStat (version 2.0; Jandel Scientific, Corte Madera, CA) was used to calculate significance. Parametric or nonparametric comparisons of data groups were performed when appropriate. The comparison used for each specific group of results is mentioned in the Results section. Differences were considered significant if $p \leq 0.05$.

\section{RESULTS}

Formation of 5-HTP from tryptophan by the enzyme tryptophan hydroxylase is thought to be the rate-limiting step in 5-HT synthesis. Bypassing this step with 5-HTP supplementation results in rapid 5-HT production through 5-HTP decarboxylation (BoadleBiber, 1982; Cooper et al., 1996). Previous work in molluscs 

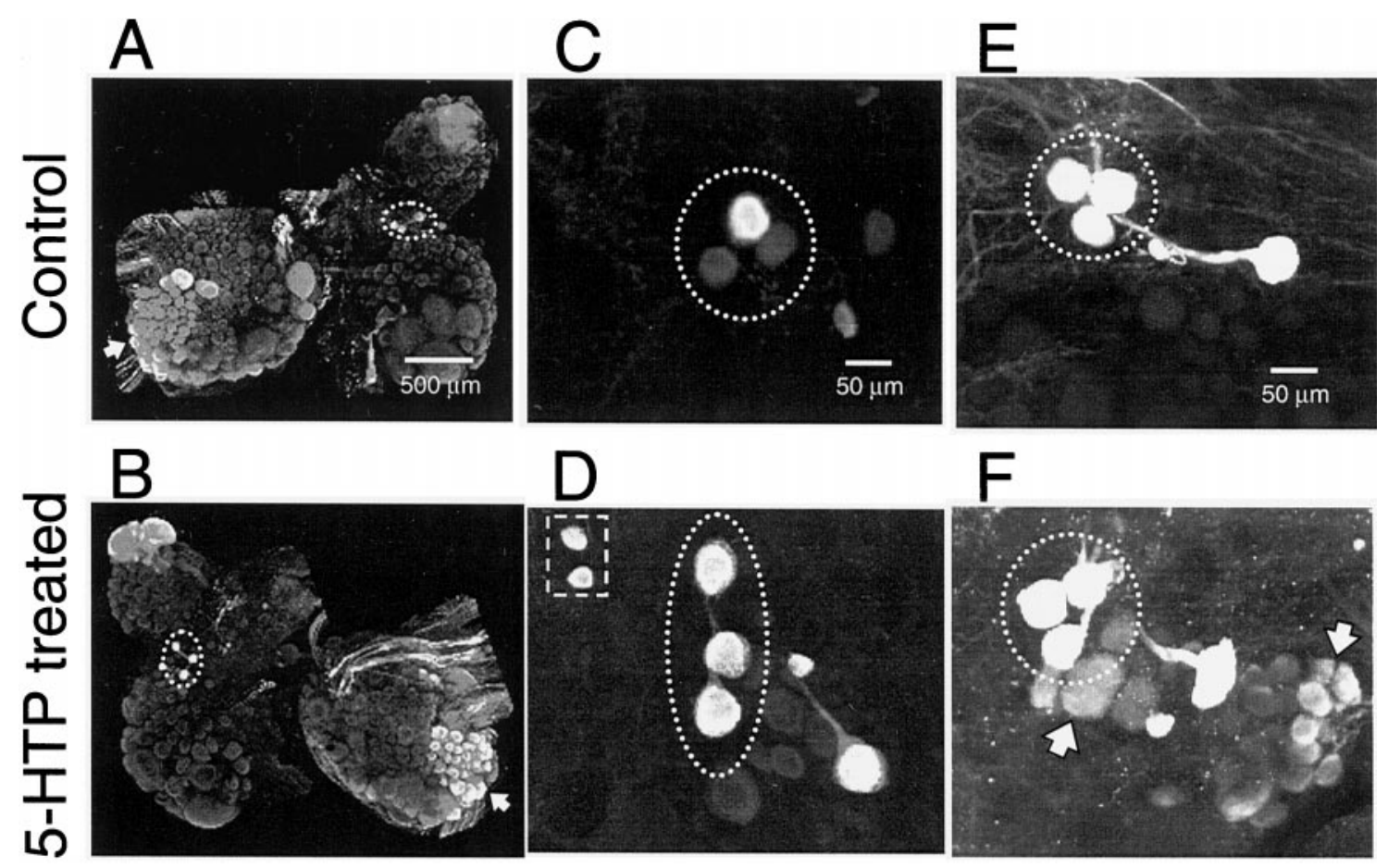

Figure 2. 5-HTP treatment increased the intensity of 5-HT immunofluorescence in Tritonia. The DSIs are circled in each image. Note that the relative orientation of the three DSIs is somewhat variable from animal to animal and even different among contralateral homologs. $A, C, E$, Untreated control preparations. $B, D, F$, Preparations treated with 5-HTP (2 mM, $60 \mathrm{~min}, 1 \mathrm{~d}$ before fixation). $A$, $B$, Comparison of 5-HT staining in the two halves of the same brain. Only the right half $(B)$ received the 5-HTP treatment, which increased the intensity of labeling in all serotonergic cells. In this illustration, the effect of 5-HTP is most apparent in the DSIs (circled), the giant serotonin cerebral cell C1 (top of image), and the posteriolateral group of pedal neurons (large groups of cells indicated by arrow). $C, D$, 5-HTP treatment increased the intensity of staining in the DSIs and caused the appearance of 5-HT immunoreactivity in a pair of neurons lateral to the DSIs $(D$, square). Note the variable level of DSI staining in the control preparation $(C)$. The 5-HT immunofluorescence is also increased in the two medial (to the right) serotonergic cells, previously identified by Sudlow et al. (1998). The preparations shown in $A-D$ were processed with 5-HT antiserum at 1:10,000 dilution. For illustration purposes, the paired images, $A$ and $B$ and $C$ and $D$, were contrast-enhanced to the same degree within each pair, to accentuate the effects of 5-HTP treatment on 5-HT immunofluorescence. $E$, $F$, Treatment with 5-HTP increased the number of neurons displaying low levels 5-HT immunoreactivity $(F$, arrows). To increase the sensitivity of the staining procedure, the antiserum dilution was decreased to 1:1000, and the amplifier gain of the photodetectors was increased, resulting in a saturation of normal 5-HT staining in the DSIs (circled). Images $C-F$ are from left cerebropleural ganglia.

demonstrated that 5-HTP can be taken up by neurons (Pentreath and Cottrell, 1972, 1973; Audesirk, 1985) and converted to 5-HT (Cottrell and Powell, 1971; McCaman et al., 1984). We used 5-HTP treatment as a means of increasing the 5-HT content of all serotonergic neurons, including the DSIs. We then examined what effect this had on DSI potency and how it altered their participation in the swim CPG.

\section{5-HTP treatment enhances serotonin immunoreactivity}

To determine whether 5-HTP treatment specifically increased the 5-HT content of serotonergic neurons or whether it caused widespread synthesis of 5-HT in normally nonserotonergic neurons, we examined the effect 5-HTP treatment on serotonin immunoreactivity. Ganglia were bathed in 5-HTP ( $2 \mathrm{~mm}$ for $30 \mathrm{~min}$ ) and then allowed to sit overnight $(20 \mathrm{hr})$ before fixing and processing for serotonin immunohistochemistry. The levels of immunofluorescence in treated brains were normalized and compared to those of untreated, control, preparations to allow comparisons across processing groups (see Materials and Methods).

5-HTP treatment increased the intensity of 5-HT immunofluorescence in all groups of serotonergic neurons, including the DSIs, the serotonergic swim CPG interneurons (Fig. 2A,B). The average normalized 5-HT immunofluorescence intensity of the DSIs increased by $32 \%$ after 5 -HTP treatment, a significant change (Table 1, Fig. 2C,D). 5-HTP treatment also increased the immunofluorescence of other serotonergic neurons, such as the neurons clustered posteriolaterally in the dorsal pedal ganglion (Fig. 2A,B, arrow). The immunofluorescence levels of these unidentified cells were measured as a group instead of individually and were normalized. 5-HTP treatment (2 mM, $30 \mathrm{~min})$ significantly increased the normalized 5-HT immunofluorescence levels in the dorsal, posterolateral group of pedal neurons by $58 \%$ (Table 1).

Most of the normally non-5-HT-immunoreactive neuronal somata remained negative for 5-HT staining after 5-HTP treatment (Fig. 2, compare $A, B$ ). However, a bilaterally represented pair of unidentified cells, located lateral to the DSIs, were not observed in untreated preparations $(n=8)$ (Fig. $2 C)$, but exhibited strong 5-HT immunoreactivity after 5-HTP treatment $(n=8)$ (Fig. 2D, square). In addition, 5-HTP treatment resulted in the appearance of 5-HT immunoreactivity in two bilaterally represented groups of neurons on the ventral surface of the brain (data not shown). One group of newly 5-HT immunoreactive neurons was located posteriolateral to the giant $\mathrm{C} 1$ neuron on the ventral side of the cerebral ganglion and consisted of $\sim 22$ cell bodies ranging in diameter from 40 to $100 \mu \mathrm{M}$. The other group of newly 5-HT immunoreactive neurons was located on the anterior ventral surface of the pedal ganglion and consisted of $\sim 40$ cell bodies, ranging in diameter from 20 to $60 \mu \mathrm{M}$. 


\begin{tabular}{|c|c|c|c|c|}
\hline & Control & 5-HTP treated & Significance & $(p)$ \\
\hline \multicolumn{5}{|l|}{ 5-HT immunofluorescence ${ }^{a}$} \\
\hline DSIs & $0.41 \pm 0.04(46)$ & $0.54 \pm 0.03(47)$ & * & $(0.01)^{\#}$ \\
\hline Pedal neurons ${ }^{b}$ & $0.57 \pm 0.03(4)$ & $0.90 \pm 0.04(4)$ & $* *$ & $(0.002)^{\dagger}$ \\
\hline \multicolumn{5}{|l|}{ DSI synaptic strength } \\
\hline DSI to DFNa (summated) ${ }^{c}$ & $7.3 \pm 1.1 \mathrm{mV}(8)$ & $14.9 \pm 2.5 \mathrm{mV}(8)$ & $* *$ & $(0.003)^{\dagger}$ \\
\hline DSI to DFNa (single) & $2.7 \pm 0.7 \mathrm{mV}(9)$ & $3.3 \pm 1.1 \mathrm{mV}(9)$ & - & $(0.3)^{\dagger}$ \\
\hline DSI to DFNa (facilitation) ${ }^{d}$ & $147 \pm 14 \%(8)$ & $272 \pm 40 \%(8)$ & $*$ & $(0.02)^{\dagger}$ \\
\hline DSI to $\mathrm{C} 2$ (summated) $^{c}$ & $0.8 \pm 0.1 \mathrm{mV}(6)$ & $2.7 \pm 0.8 \mathrm{mV}(6)$ & $*$ & $(0.03)^{\ddagger}$ \\
\hline \multicolumn{5}{|l|}{ DSI modulation of $\mathrm{C} 2$} \\
\hline $\mathrm{C} 2$ excitability $^{e}$ & $6 \pm 3 \%(6)$ & $28 \pm 5 \%(6)$ & $* *$ & $(0.002)^{\dagger}$ \\
\hline C2 synaptic strength ${ }^{f}$ & $51 \pm 23 \%(7)$ & $112 \pm 32 \%(7)$ & $*$ & $(0.03)^{\ddagger}$ \\
\hline \multicolumn{5}{|l|}{ Nerve-evoked swim CPG activity } \\
\hline DSI, \#APs/CPG activity ${ }^{g}$ & $133 \pm 15(15)$ & $34 \pm 6(15)$ & $* *$ & $(0.001)^{\dagger}$ \\
\hline $\mathrm{C} 2, \# \mathrm{APs} / \mathrm{CPG}$ activity & $165 \pm 19(9)$ & $182 \pm 21(9)$ & - & $(0.5)^{\dagger}$ \\
\hline Duration of CPG activity & $36 \pm 4 \sec (15)$ & $48 \pm 5 \sec (15)$ & $*$ & $(0.008)^{\dagger}$ \\
\hline Electrochemical signal & $0.17 \pm 0.05 \mathrm{ACU}^{h}(6)$ & $0.46 \pm 0.07 \mathrm{ACU}(6)$ & * & $(0.02)^{\dagger}$ \\
\hline
\end{tabular}

All values are given as mean \pm SEM, with number of observations listed in parentheses.

*Denotes significant change from control $(p \leq 0.05)$; **indicates highly significant change $(p \leq 0.005)$. $p$ values are listed in parentheses in the last column.

${ }^{a}$ Normalized intensity.

${ }^{b}$ The dorsal, posterolateral group of serotonergic neurons in the pedal ganglia.

${ }^{c}$ Five pulses at $5 \mathrm{~Hz}$.

${ }^{d}$ Percent increase in amplitude of third EPSP compared to first EPSP, evoked at $5 \mathrm{~Hz}$.

${ }^{e}$ Percent increase in average instantaneous spike frequency following DSI activity.

${ }^{f}$ Percent increase in amplitude of EPSP evoked by C2 in DFNa after DSI activity.

${ }^{g} \mathrm{CPG}$ activity, nerve-evoked activity.

${ }^{h} \mathrm{ACU}$, Arbitrary concentration units.

See Materials and Methods and Results for further details of experiments. ${ }^{\#}$ Unpaired $t$ test. ${ }^{\dagger}$ Paired $t$ test. ${ }^{\star}$ Wilcoxon signed rank test.

We also found an increase in the consistency of low-intensity 5-HT immunolabeling of neurons, near the DSIs, that had been previously reported to express a low level of 5-HT immunoreactivity (Sudlow et al., 1998) (Fig. 2E,F). Under conditions that maximized staining (high primary antiserum concentration and increased gain on the photodetectors), we did not observe any staining in these cells in 8 of 13 control preparations (Fig. 2E). However, under identical staining conditions, these cells were observable in all of the 13 5-HTP-treated preparations examined (Fig. 2F).

\section{5-HTP does not have acute, short-term actions}

We examined whether 5-HTP acted purely as synthesis precursor or whether it also had direct effects on 5-HT receptors. Application of 5-HTP (1-2 mM) did not evoke any immediate actions on neurons within the swim system, as monitored electrophysiologically. There were no noticeable changes in either the resting membrane potentials or non-swim firing rates of the DSIs and other swim interneurons during the 30-60 min of 5-HTP application $(n=13)$. By contrast, 5-HT $\left(10^{-4} \mathrm{M}\right)$ depolarized DSI and $\mathrm{C} 2$, causing them to fire APs within 5 min of the start of bath application ( $n=2$; data not shown). Additionally, we observed that within 6-8 min, 5-HT triggered rhythmic swim CPG activation, as previously reported (McClellan et al., 1994), whereas 5-HTP never triggered rhythmic activity during exposures of up to $60 \mathrm{~min}$. Nor did 5-HTP block nerve stimulation from evoking a swim motor program, indicating that, at the concentrations used, 5-HTP does not act as an agonist or antagonist of serotonin receptors.

\section{Treatment with 5-HTP increases the amplitude of DSI-evoked synaptic potentials}

The effect of 5-HTP treatment on the strength of DSI-evoked monosynaptic serotonergic EPSPs recorded in postsynaptic followers was examined. As expected, 5-HTP treatment (1 or $2 \mathrm{~mm}$, 30-60 $\mathrm{min}$ ) enhanced the subsequent potency of the DSIs at evoking synaptic actions (Fig. 3). One day after 5-HTP treatment, the summated fast EPSP recorded in a dorsal flexion neuron (DFNa) in response to DSI stimulation (five pulses at $5 \mathrm{~Hz}$ ) was $204 \%$ of the control value, a highly significant increase (Table 1). 5-HTP treatment also significantly increased the amplitude of the summated EPSPs evoked by the DSIs in another CPG interneuron, $\mathrm{C} 2$, by $238 \%$ (Table 1). In untreated preparations recorded over similar periods, DSI-evoked EPSPs never increased in amplitude.

The increase in DSI fast synaptic actions appears to be attributable to an enhancement of homosynaptic facilitation rather than an increase in the amplitude of single DSI-evoked EPSPs. Individually evoked EPSPs recorded in a DFNa were not significantly different in amplitude after 5-HTP treatment (Table 1). However, the degree of facilitation (see Materials and Methods) of EPSPs evoked in DFNa by DSI firing at $5 \mathrm{~Hz}$ significantly increased; the amplitude of the third EPSP, relative to the first EPSP, increased by $85 \%$ (Table 1). Thus, the effect of 5-HTP treatment was only apparent when examining EPSPs evoked by DSI spike trains: greater facilitation resulted in larger summated EPSPs and caused DFNa to reach AP threshold during prolonged DSI stimulation (Fig. 3B). 

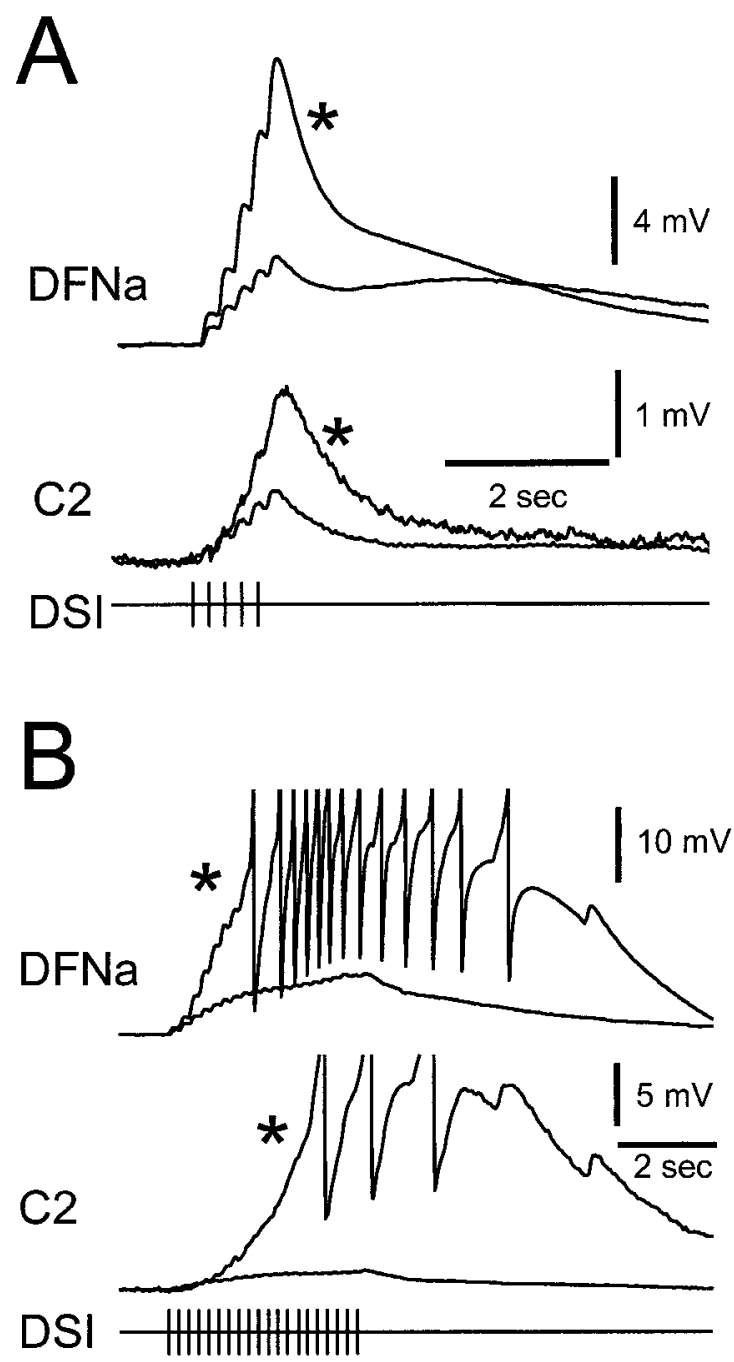

Figure 3. The synaptic effects of DSI were enhanced $1 \mathrm{~d}$ after treatment with 5-HTP. A, Simultaneous intracellular recordings from a dorsal flexion neuron $(D F N a)$ and cerebral interneuron $\mathrm{C} 2$ before and after $(*)$ 5-HTP treatment $(1 \mathrm{mM}, 30 \mathrm{~min})$ show an increase in the size of EPSPs evoked by DSI stimulation $(5 \mathrm{~Hz}, 1 \mathrm{sec}) . B$, 5-HTP treatment (*) resulted in superthreshold responses to a longer duration $5 \mathrm{~Hz}$ DSI spike train. DFNa membrane potential was maintained at $-70 \mathrm{mV}$ before DSI stimulation with current injection in two-electrode current-clamp mode. Despite the fact that all recordings in this figure were made in high divalent cation saline to suppress polysynaptic communication, some recruited EPSPs are apparent in the 5-HTP traces in $B$.

\section{5-HTP treatment enhances the strength of DSI neuromodulatory actions}

In addition to classical synaptic actions, DSI stimulation also evokes two neuromodulatory actions that have been attributed to release of 5-HT: enhancement of C2 excitability and presynaptic facilitation of C2 synaptic strength (Katz et al., 1994; Katz and Frost, 1995a,b, 1997). Neither modulatory effect was observed to increase over time in untreated preparations. We tested whether 5-HTP treatment augmented the neuromodulatory potency of the DSIs.

To test the effect of 5-HTP treatment on the ability of the DSIs to enhance $\mathrm{C} 2$ excitability, we chose stimulation parameters that resulted in a very small neuromodulatory effect under control conditions (Fig. $4 A$ ). Before treatment, DSI stimulation $(5 \mathrm{~Hz}, 3$ $\mathrm{sec}$ ) did not significantly increase the average instantaneous spike frequency (ISF) of C2 (Fig. 4C). However, $20 \mathrm{hr}$ after 5-HTP treatment, although baseline $\mathrm{C} 2$ excitability was higher, the same DSI stimulus now produced a further significant enhancement of C2 excitability (Table 1, Fig. 4B-D). The increased basal C2 excitability after 5-HTP treatment may be attributable to a direct effect of 5-HTP exposure or an increase in tonic 5-HT release from serotonergic cells in the brain.

Serotonin released from the DSIs also modulates C2 synaptic strength, acting presynaptically to enhance transmitter release (Katz et al., 1994; Katz and Frost, 1995a,b). We examined the effect of 5-HTP treatment on C2-evoked EPSPs recorded in the postsynaptic follower DFNa (see Materials and Methods). Using DSI stimulation parameters $(5 \mathrm{~Hz}, 10 \mathrm{sec})$ previously used for demonstrating DSI modulation of C2 synapses, 5-HTP treatment increased the extent and duration of the DSI modulatory action, causing a large increase in the size of C2-evoked synaptic potentials (Fig. 5) $(n=2)$. As previously reported, under control conditions (Fig. 5A,C) the modulatory actions dissipated by 20 sec after a DSI stimulus. However, after 5-HTP treatment, there was still a robust enhancement of $\mathrm{C} 2$ synaptic strength even $1 \mathrm{~min}$ after DSI stimulation (Fig. $5 B, C$ ).

Although 5-HTP increased the effectiveness of DSI stimulation at enhancing $\mathrm{C} 2$ synaptic strength, it is unlikely that the result shown in Figure 5 is attributable entirely to a direct action of the DSI on C2; clearly other neurons were recruited by the DSI stimulus. We therefore switched to a shorter $(1 \mathrm{sec})$, less effective DSI stimulus train to examine the effect of 5-HTP on this modulatory action. Under control conditions this DSI stimulus had a more modest and variable effect on the size of C2-evoked synaptic potentials, enhancing them on average by $51 \pm 23 \%$. After 5 -HTP treatment, DSI stimulation enhanced $\mathrm{C} 2$-evoked responses by $112 \pm 32 \%$, a significant increase over control conditions (Table 1).

\section{5-HTP treatment enhances the DSI potency at evoking rhythmic CPG activity}

Previously, it was reported that tonic depolarization of C2 could elicit a rhythmic swim motor program (Getting, 1977; Taghert and Willows, 1978), but tonic depolarization of one or more DSIs was not sufficient to trigger a swim motor program from a rested state (Lennard et al., 1980). Based on the known organization of the swim network, with the DSIs being the sole recipient of synaptic input from the dorsal ramp interneuron (DRI) (Fig. 1 $A$ ), it seemed that DSI activity ought to be sufficient to trigger the swim motor program. However, depolarization of one or more DSIs with a constant current pulse never evoked prolonged bursting activity in untreated preparations (Fig. 6 $\mathrm{A}$ ).

The reason that constant current depolarization of the DSIs was insufficient to trigger a rhythmic motor pattern may be because this method of excitation did not cause the DSIs to fire action potentials at a sufficiently high frequency. The DSIs exhibit spike frequency adaptation, which limits their ability to fire rapidly for prolonged periods of time. Furthermore, each DSI recruits inhibition onto itself and onto the other DSIs from a set of, as yet, unidentified interneurons (Fig. 1A) (Getting et al., 1980; Getting and Dekin, 1985). However, these self-limiting features can be overridden when a DSI is driven to spike tonically using brief current pulses (see Materials and Methods). Firing a single DSI at $5-20 \mathrm{~Hz}$ in this manner, it was often possible to evoke a bursting motor program. The periodicity of this rhythmic bursting pattern was very similar to the nerve-evoked swim motor program. Unlike with nerve stimulation however, there was a long latency to the first burst after the start of DSI firing $(25 \pm 2 \mathrm{sec}$; 

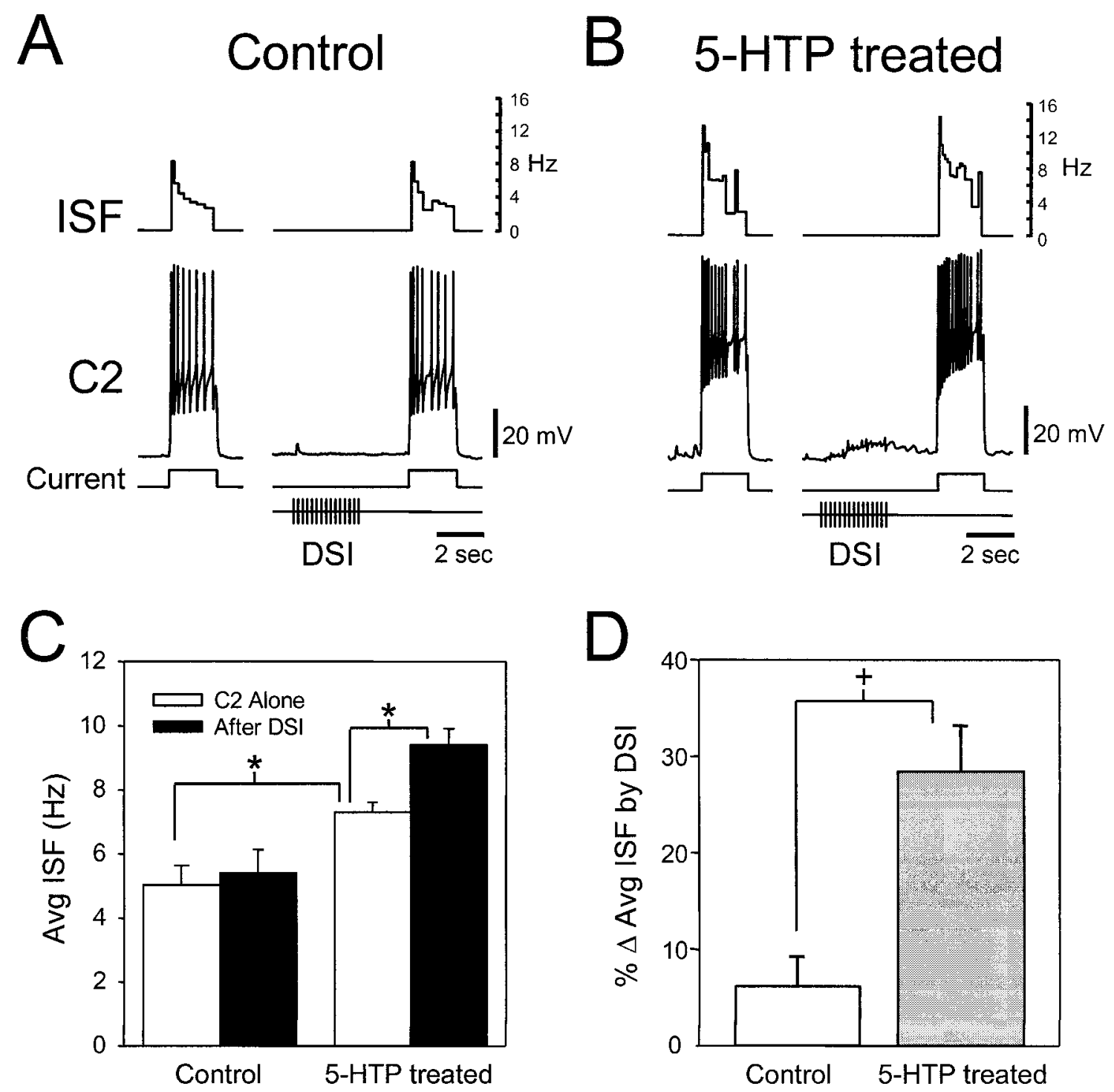

Figure 4. 5-HTP treatment increases $\mathrm{C} 2$ excitability and the potency of DSI neuromodulation. $A$, Under conditions designed to limit swim CPG activity, DSI has little effect on $\mathrm{C} 2$ excitability. C2 excitability is quantified as the spiking response to a 2 sec 2 nA depolarizing current pulse applied every 60 sec. The inverse interspike interval or instantaneous spike frequency (ISF) is displayed in the top trace. Stimulation of a DSI (15 pulses at $5 \mathrm{~Hz}$, ending $2 \mathrm{sec}$ before $\mathrm{C} 2$ depolarization) has no significant effect on the spiking response of $\mathrm{C} 2$. B, After 5-HTP treatment $(2 \mathrm{~mm}, 30 \mathrm{~min}, 20 \mathrm{hr}$ before measurement), the same stimulation paradigm results in an increase in basal C2 excitability and a further enhancement of C2 excitability by DSI. $C$, 5-HTP treatment enhances the average basal C2 ISF and its modulation by DSI. Under control conditions, the average ISF was not significantly different if C2 was stimulated alone (white bar) or if preceded by DSI stimulation (black bar). After 5-HTP treatment, the average C2 ISF increased significantly when $\mathrm{C} 2$ was stimulated alone. A further significant increase was seen when $\mathrm{C} 2$ was depolarized after DSI stimulation. The asterisk indicates a significant difference ( $p<0.05$, one-way repeated measures ANOVA with Tukey post hoc test; $n=6)$. $D$, 5-HTP treatment significantly increases DSI neuromodulatory enhancement of C2 average ISF ( $p=0.002, n=6$; paired $t$ test). The data in $D$ represent a further analysis of the data shown in $C$ and depict the enhancement of C2 average ISF ( $\% \Delta$ Avg ISF) by DSI activity before (Control) and 20 hr after 5-HTP treatment (5-HTP treated). All recordings were obtained in normal saline.

$n=12$ ) (Fig. $6 B$ ). Also, unlike the swim motor program elicited by nerve stimulation, which continues for up to a minute after the end of the stimulus, the rhythmic bursting activity was not sustained for more than one cycle after the suspension of DSI stimulation.

After 5-HTP treatment, the ability of the DSIs to elicit prolonged rhythmic activity was dramatically increased. Now, brief depolarization of one DSI could trigger a prolonged depolarization of the $\mathrm{C} 2$ neurons and rhythmic bursts of APs that far outlasted the DSI activity $(n=5)$ (Fig. $6 C$ ). Although the bursting pattern again had a similar frequency to the nerve-evoked swim motor program, there were distinct differences in the ap- pearance of the bursts. Most noticeably, during these prolonged bouts of DSI-triggered activity, the DSIs themselves did not fire rhythmic bursts of APs, and the C2 bursts were punctuated by hyperpolarizations that were weaker than those in the nerveevoked swim motor program.

\section{The activity patterns of swim CPG neurons are altered by 5 -HTP treatment}

Given that the DSIs are components of the swim CPG circuit and that their synaptic and modulatory actions are greatly enhanced by 5-HTP treatment, we wanted to know what effect 5-HTP treatment has on nerve-evoked swim CPG activity. One day after 


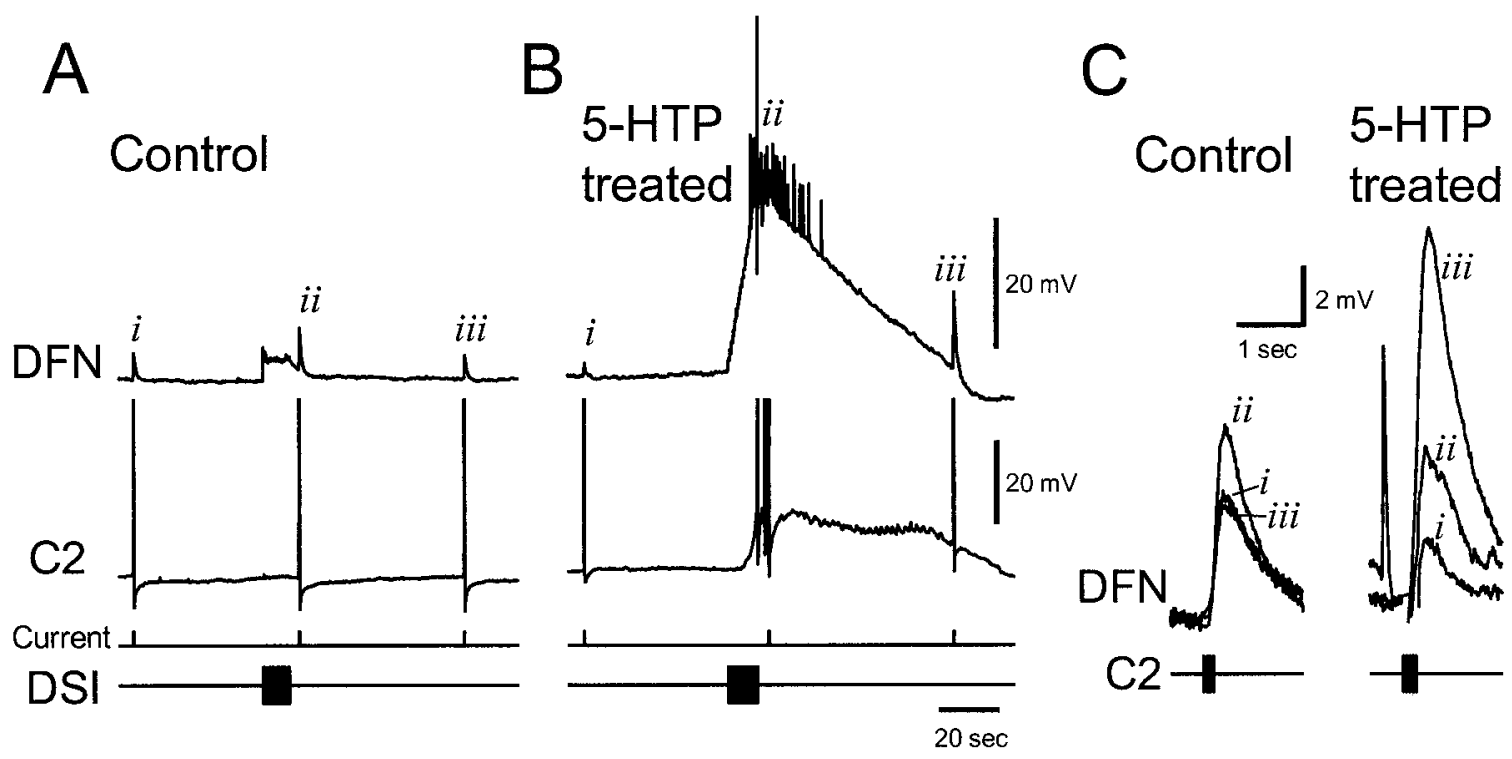

Figure 5. 5-HTP treatment increases DSI neuromodulation of $\mathrm{C} 2$ synaptic strength. $A$, Under control conditions, DSI enhanced the C2-evoked summated EPSP in a dorsal flexion neuron, DFN, when C2 was stimulated $3 \mathrm{sec}$ after the end of the AP train in DSI. But $60 \mathrm{sec}$ after that, the EPSP was back to pre-DSI levels. B, After 5-HTP treatment (1 mM, $40 \mathrm{~min}, 24 \mathrm{hr}$ before recording), an AP train in DSI evokes a much larger response in C2 and the DFN. The C2-evoked EPSP in DFN, while increased $3 \mathrm{sec}$ after DSI stimulation (but not discernible in the midst of the myriad polysynaptic EPSPs and one truncated AP; however, see part $C$ ), is even larger $60 \mathrm{sec}$ later. $C$, Faster timescale depictions of the summated C2-evoked EPSPs from the control and 5-HTP-treated recordings show the long-lasting modulation of C2 synaptic strength by DSI. The overlapped traces from control and 5-HTP-treated conditions correspond to the summated EPSPs, denoted by $i, i i$, and $i i i$ in $A$ and $B$, respectively. The summated EPSPs have been baselineadjusted for comparison. The additional fast EPSP in the 5-HTP-treated group immediately preceded summated EPSP $i i$ (from $B$ ) and originated from some cell other than the C2 or DSI. For both control and 5-HTP-treated conditions, the DSI was stimulated at $2 \mathrm{~Hz}$ for $10 \mathrm{sec}$. The C2 was stimulated to fire four APs at $20 \mathrm{~Hz}$ for the control and five APs after 5-HTP treatment. The number of APs fired by C2 was increased to facilitate measurement of the summated EPSP.
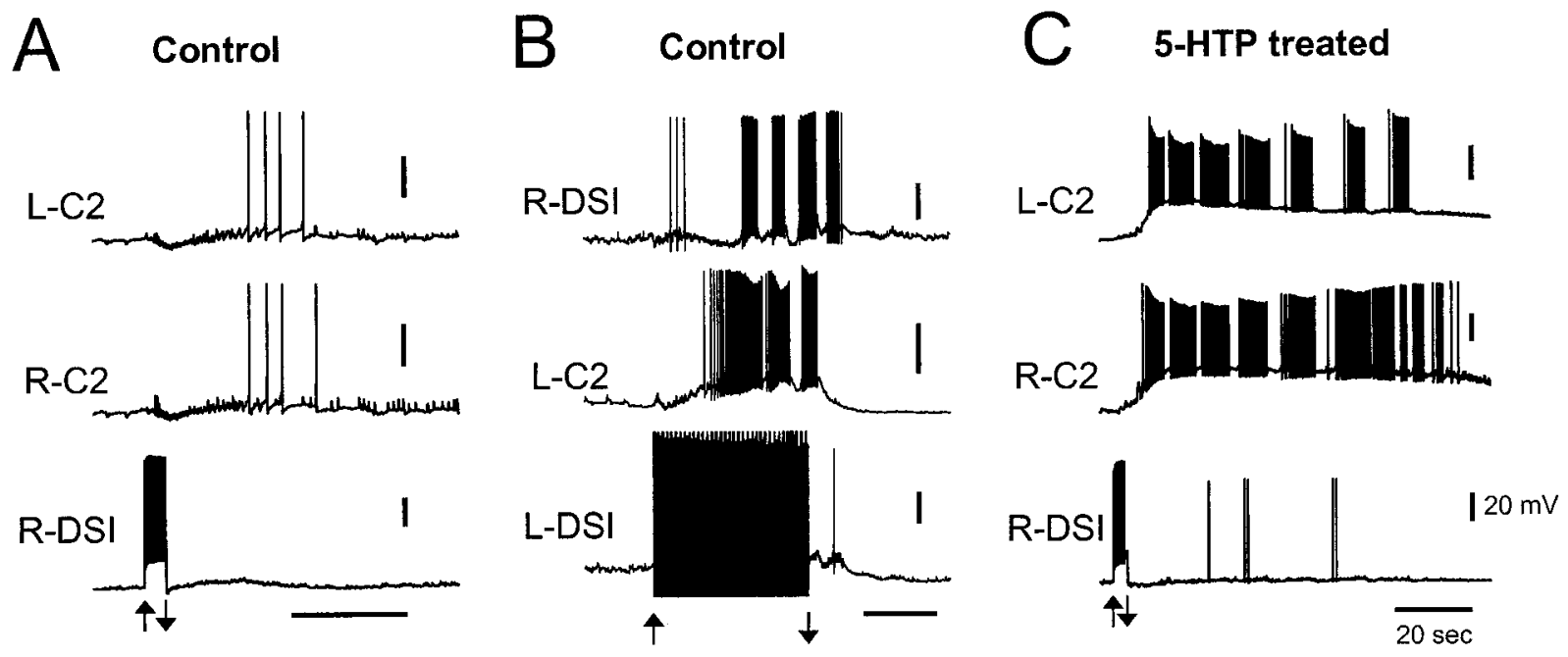

Figure 6. Treatment with 5-HTP greatly enhanced the ability of the DSIs to trigger a rhythmic swim-like motor program. A, In a control preparation, injection of a brief depolarizing current pulse $(2 \mathrm{nA}, 4 \mathrm{sec})$ into a DSI did not evoke sustained rhythmic activity in other CPG neurons. The DSI reached an average firing rate of $10 \mathrm{~Hz}$. This example represents the largest effect of brief DSI stimulation seen: DSI recruited synaptic inputs to C2 and caused a delayed period of weak firing. $B$, In a control, untreated preparation, making a single DSI fire tonically at $10 \mathrm{~Hz}$ enabled rhythmic bursting in other CPG neurons that resembled a swim motor program. The DSI was made to fire at a constant frequency through injection of repeated brief current pulses (20 msec duration, $8 \mathrm{nA}$ ); each elicited a single DSI AP. The individual APs are obscured by stimulus artifacts. $C$, One day after 5-HTP treatment, injection of a brief depolarizing current pulse $(1.5 \mathrm{nA}, 3 \mathrm{sec})$ into a DSI was sufficient to trigger a sustained period of rhythmic activity in the C2 sec (same preparation and cells as in $A$ ). The firing rate of the DSI did not exceed $5 \mathrm{~Hz}$, and the DSI did not continue to fire rhythmically during the C2 bursting as it would during a nerve-evoked swim motor program in an untreated preparation. All recordings were obtained in normal saline. For each case, the overall stimulation of the DSI starts at the up arrow and ends at the down arrow. Calibration: $20 \mathrm{mV}, 20 \mathrm{sec}$.

5-HTP treatment, there was a profound and persistent change in the CPG activity in all 17 preparations examined. Before 5-HTP treatment, nerve stimulation typically evoked motor programs that consisted of two to eight cycles of bursting activity in all of the swim CPG interneurons (Fig. 7A) (Getting, 1989a). When observed $20 \mathrm{hr}$ or more after 5-HTP treatment, cyclic bursting activity was absent in the DSIs, yet, paradoxically, general activity in the neural circuit remained high (Fig. 7B). Instead of display- 

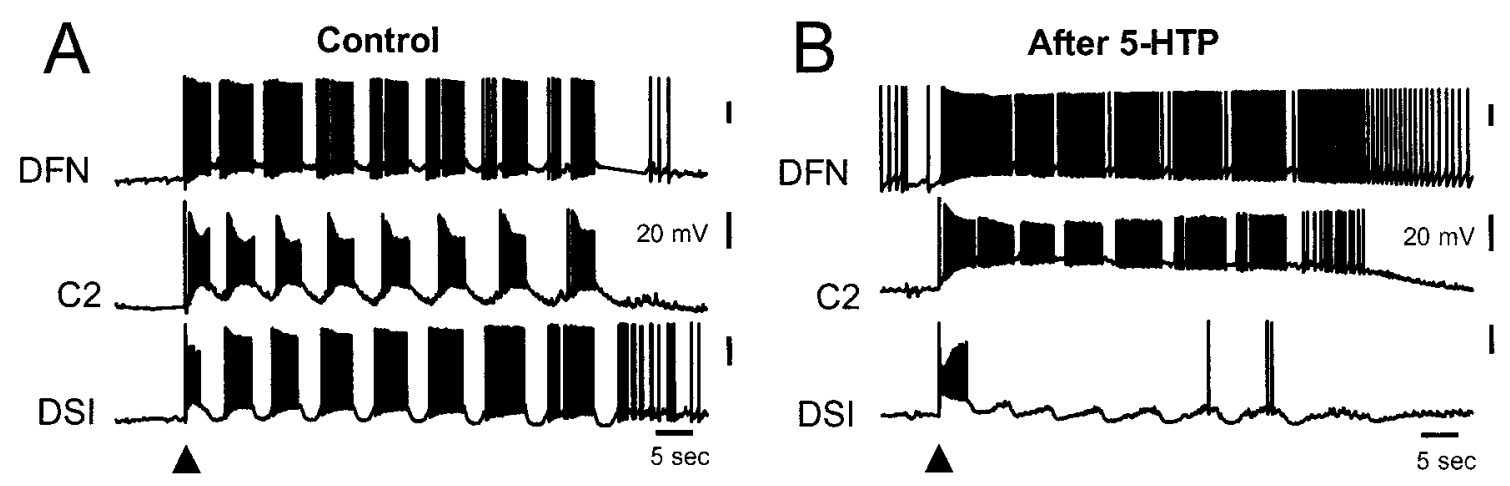

Figure 7. 5-HTP treatment greatly changes the nerve-evoked output of the swim CPG. $A$, Under control conditions, stimulation of pedal nerve 3 (10 $\mathrm{Hz}, 1 \mathrm{sec}$ at arrowhead) evoked an eight-cycle swim motor program. Rhythmic bursting activity was recorded simultaneously in a DSI, C2, and a DFN. $B$, Twenty hours after 5-HTP treatment, the same nerve stimulus (arrowhead) evoked a very different pattern of activity. C2 was strongly excited and fired bursts of action potentials without the pronounced interburst hyperpolarizations seen under control conditions. After 5-HTP treatment, the DSI fired only a single burst of APs in response to nerve stimulation and fell silent while C2 remained depolarized and bursting. Vertical scale bars are 20 $\mathrm{mV}$ for all traces. All recordings were obtained in normal saline.

ing their typical pattern of activity, the DSIs fired a single brief burst of APs after the nerve stimulus and then were silent or fired sporadically in a nonbursting fashion $(n=17)$. Thus, nerveevoked DSI spiking activity decreased $74 \%$ after treatment (Table 1). Despite the decrease in DSI spiking, the average length of nerve-evoked swim CPG output from other cells significantly increased by $33 \%$ after 5-HTP treatment (Table 1). It is highly likely that all six of the DSIs exhibited this change in behavior because it was observed for each of the DSIs from which we recorded. The change in DSI firing is not attributable to the duration of the recording session; in untreated preparations, the DSIs continued to exhibit a normal cycle-by-cycle participation in nerve-evoked swim CPG activity for at least $2 \mathrm{~d}$ in vitro $(n=10)$.

The effects of 5-HTP treatment on nerve-evoked C2 activity differed greatly from those on DSI. In approximately half of 5-HTP-treated preparations observed $20 \mathrm{hr}$ after treatment, nerve-elicited C2 activity consisted of a long-lasting depolarization accompanied by sustained AP generation and was not rhythmic (Fig. 8D). However, in 7 of 16 preparations the $\mathrm{C} 2$ firing pattern continued to display some rhythmicity $20 \mathrm{hr}$ or more after 5-HTP exposure (Fig. $7 B$ ). In those cases, the periodic hyperpolarizations corresponding to the ventral flexion phase of the motor pattern were greatly reduced. In contrast to its effect on nerve-evoked DSI spike number, the number of APs elicited in C2 was unchanged after 5-HTP treatment (Table 1).

We found that treatment with 5-HT $\left(10^{-4} \mathrm{M}\right)$ had an effect on subsequent nerve-evoked swim CPG activity that was similar to that produced by treatment with 1-2 mM 5-HTP, including disruption of the rhythmicity of nerve-evoked activity and the lack of DSI firing after an initial burst ( $n=2$; data not shown). This pattern of activity may be attributable to supplementation of neuronal 5-HT through uptake of the exogenous 5-HT and release after subsequent stimulation.

\section{The effects of 5-HTP emerge gradually}

As might be expected for a synthesis precursor, the effects of 5-HTP on the motor program became more evident over time (Fig. 8). After $30 \mathrm{~min}$ in the presence of 5-HTP, the DSIs continued to fire action potentials on every cycle of the motor program (Fig. $8 B$ ), but the number of APs per burst decreased from $27.9 \pm 4.8$ under control conditions to $17.6 \pm 4.4(p=0.03$, paired $t$ test; $n=4)$. Within 1 hr after 5-HTP washout, the DSIs generally fired only a single burst of APs in response to nerve stimulation and then fell silent or participated minimally for the remainder of the stimulated swim CPG output (Fig. 8C). The DSIs continued to display this reduced activity in response to nerve stimulation for at least $2 \mathrm{~d}$ after treatment (longer times were not tested). Although the DSIs progressively decreased their spike frequency and eventually ceased to burst, C2 bursting and the length of the average swim CPG output were not noticeably affected until many hours after 5-HTP washout (Table 1, Fig. 8D).

\section{5-HTP treatment increases the size of electrochemically measured signals evoked by nerve stimulation}

As we have shown, 5-HTP treatment had competing effects: it enhanced the synaptic and neuromodulatory potency of the DSIs and their ability to evoke rhythmic activity from the circuit, yet it also decreased DSI spiking activity during nerve-evoked CPG activation. This raises the question of whether 5-HTP treatment changes the net amount of 5-HT released in response to nerve stimulation. We used microvoltammetry to measure the influence of 5-HTP pretreatment on the electrochemical signal evoked by nerve stimulation. Oxidation and reduction currents characteristic of 5-HT were recorded using Nafion-coated carbon fiber electrodes in response to nerve stimuli that evoked swim motor programs. The carbon fiber was inserted into the neuropil in a lateral region of the cerebral ganglion that putatively contains DSI arborizations (Fig. 1C, arrows; Getting et al., 1980, their Fig. 1). Signals could be obtained only in restricted areas, presumably corresponding to sites of 5-HT release.

Pretreatment with 5-HTP increased the amplitude of nervestimulated putative 5-HT overflow at these sites (Fig. 9). The average size of detectable signals before 5-HTP pretreatment was $0.17 \pm 0.05$ ACU (see Materials and Methods). This was significantly increased by $171 \%$ to $0.46 \pm 0.07 \mathrm{ACU}$ at those same sites after 5-HTP exposure (Table 1). These results are consistent with an increase in 5-HT release induced by precursor pretreatment. Although the DSIs fired less, the size of the putative 5-HT signal elicited by nerve stimulation was greater. It is possible, therefore, that the signal originates from activity of serotonergic neurons in addition to the DSIs.

\section{The behavioral effects of 5-HTP injection}

The effects of 5-HTP treatment on swim CPG activity in Tritonia may represent an extreme example of the "serotonin syndrome" 

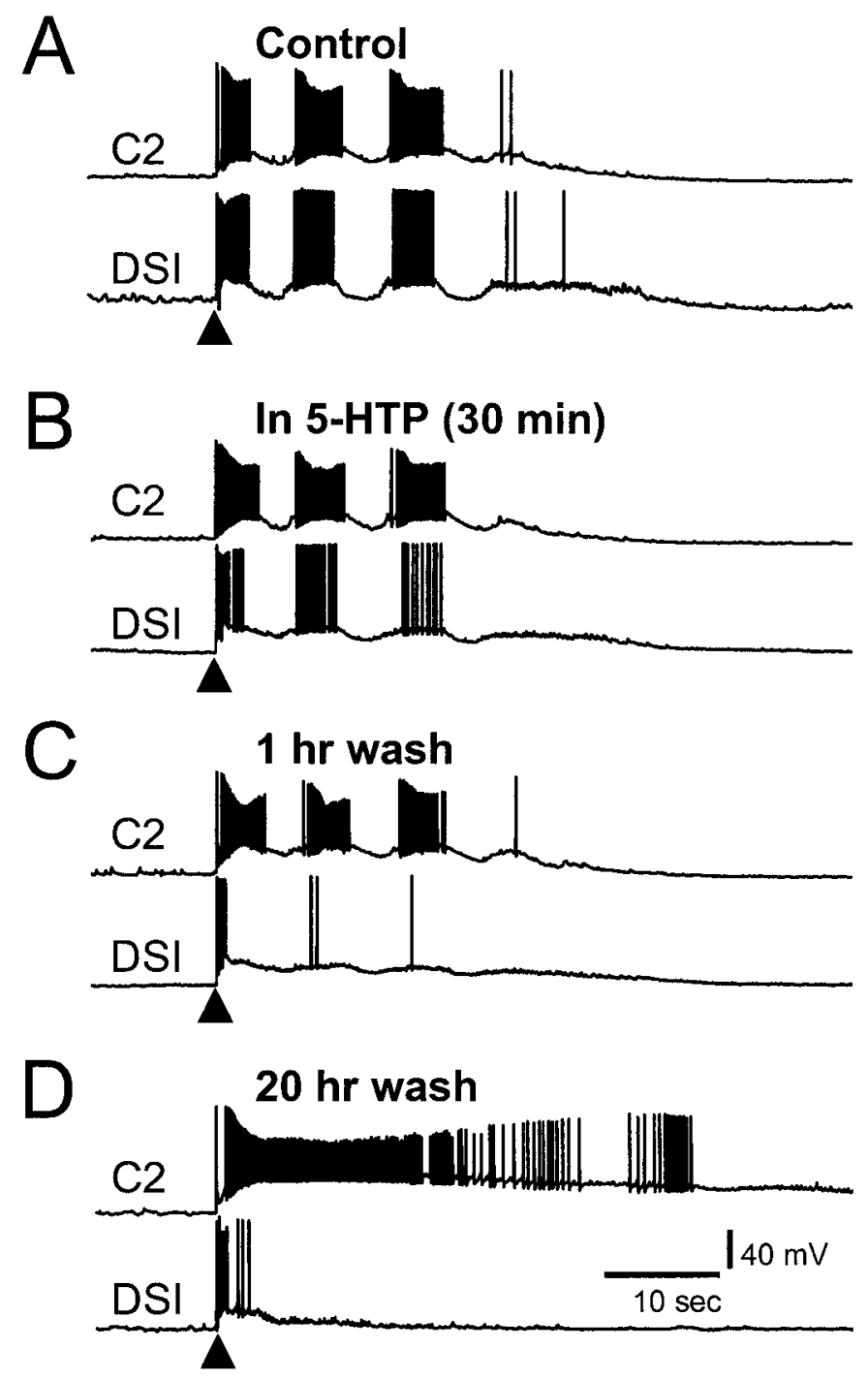

Figure 8. The effects of 5-HTP emerge gradually even after 5-HTP washout. $A$, Before treatment, pedal nerve 3 stimulation $(10 \mathrm{~Hz}, 1 \mathrm{sec}$ at arrowhead) elicited a swim motor program consisting of three dorsal flexion cycles. $B$, Thirty minutes after the start of $2 \mathrm{mM} 5-\mathrm{HTP}$, the DSI fired fewer APs per burst, whereas $\mathrm{C} 2$ firing was unchanged. $C$, One hour after the end of a $30 \mathrm{~min} 5$-HTP application, the cycle-by-cycle DSI bursting was largely diminished, but $\mathrm{C} 2$ bursting was still unchanged. $D$, By $20 \mathrm{hr}$ after 5-HTP treatment, nerve-evoked output of the circuit was at its final form. The DSI fired only a brief initial burst of APs in response to nerve stimulation, whereas $\mathrm{C} 2$ exhibited a sustained period of intense firing uninterrupted by hyperpolarizing periods. Recordings were obtained in normal saline.

induced in vertebrates by serotonergic drugs that produce excessive 5-HT levels. Serotonin syndrome consists of a variety of motor symptoms, including myoclonus, tremor, and rigidity in mammals (Sternbach, 1991; Jacobs and Fornal, 1997). Observations of intact Tritonia after 5-HTP injection $(10 \mathrm{mg} / 100 \mathrm{gm}$ body weight) show a radical alteration in behavior presumably attributable to an enhancement of 5-HT throughout the CNS and periphery. 5-HTP injection, unlike control, vehicle injections, evoked periods of prolonged dorsal flexion several hours after injection, and subsequently interfered with swim production, reducing the intensity and range of the dorsal flexion component of the swim, for a period of $2-3 \mathrm{~d}$ ( $n=3$; data not shown).

\section{DISCUSSION}

Our findings illustrate that 5-HT supplementation by precursor treatment can have unexpected results for neural circuit function and interrupt motor pattern generation in the nervous system. Although 5-HTP pretreatment increased DSI potency in evoking synaptic and neuromodulatory actions and in triggering prolonged bursting of other CPG neurons, their spiking activity during swim circuit operation was reduced to a single burst at the onset of the motor program. Despite the massive reduction in DSI activity, the excitation of other cells in the circuit, particularly C2, remained high, and a larger electrochemical signal was recorded. Thus, more serotonin did not result in a more robust motor program.

\section{5-HTP enhancement of serotonergic actions}

Treatment of the nervous system resulted in an increase in the potency of DSI effects. The DSIs evoke fast and slow EPSPs as well as neuromodulatory actions. Previous work suggested that these actions are all mediated by 5 -HT release (Katz and Frost, 1995b). Enhancement of these DSI-evoked effects by 5-HTP treatment is further evidence that the DSIs release 5-HT.

The mechanism underlying the 5-HTP enhancement of DSI synaptic potentials may yield insight into the serotonin release process. The availability of releasable transmitter may play a role in the ability of synapses to exhibit facilitation in other systems (Schlapfer et al., 1974; Katz et al., 1993; Verhage et al., 1994). In this system, 5-HTP treatment did not significantly increase the size of single EPSPs evoked by DSI, but it strongly enhanced the amount of synaptic facilitation. This may indicate that basal release is not limited by available 5-HT. In contrast, synaptic facilitation caused by residual calcium buildup (Zucker, 1989) may normally deplete the store of readily releasable 5-HT and may thus be enhanced by increasing 5-HT synthesis.

\section{Serotonin released from the DSIs can trigger the swim motor program}

The DSIs are the only known CPG neurons to receive synaptic input from the escape swim command neuron, the DRI (Frost and Katz, 1996). Because this pathway initiates the swim response, DSI activation should be sufficient to elicit the swim motor program. Previous work established that 5-HT is necessary for swim motor program production, because 5-HT antagonists block production of the swim (McClellan et al., 1994). Although application of exogenous 5-HT elicits a swim motor program (McClellan et al., 1994), it had not been previously shown that the DSIs serve as a sufficient source of 5-HT to activate the swim motor program.

The DSIs recruit strong inhibition onto themselves (Getting et al., 1980; Getting and Dekin, 1985). Therefore, sustained depolarization of one DSI inhibits the other DSIs, decreases firing in the depolarized cell, and does not evoke rhythmic activity. To circumvent recurrent inhibition, we drove APs in single DSIs with repetitive current pulses, thus maintaining a constant firing rate. This evoked rhythmic activity in other swim interneurons (including other DSIs) that resembled the swim motor program in its periodicity, indicating that DSI activation is sufficient to elicit the swim motor program. It should be noted that although the DSIs were stimulated to fire tonically, they received rhythmic synaptic feedback that might have gated their synaptic output and transformed tonic firing into a rhythmic synaptic output.

After 5-HTP treatment, the ability of DSIs to elicit rhythmic activity in swim interneurons was greatly enhanced. Instead of 

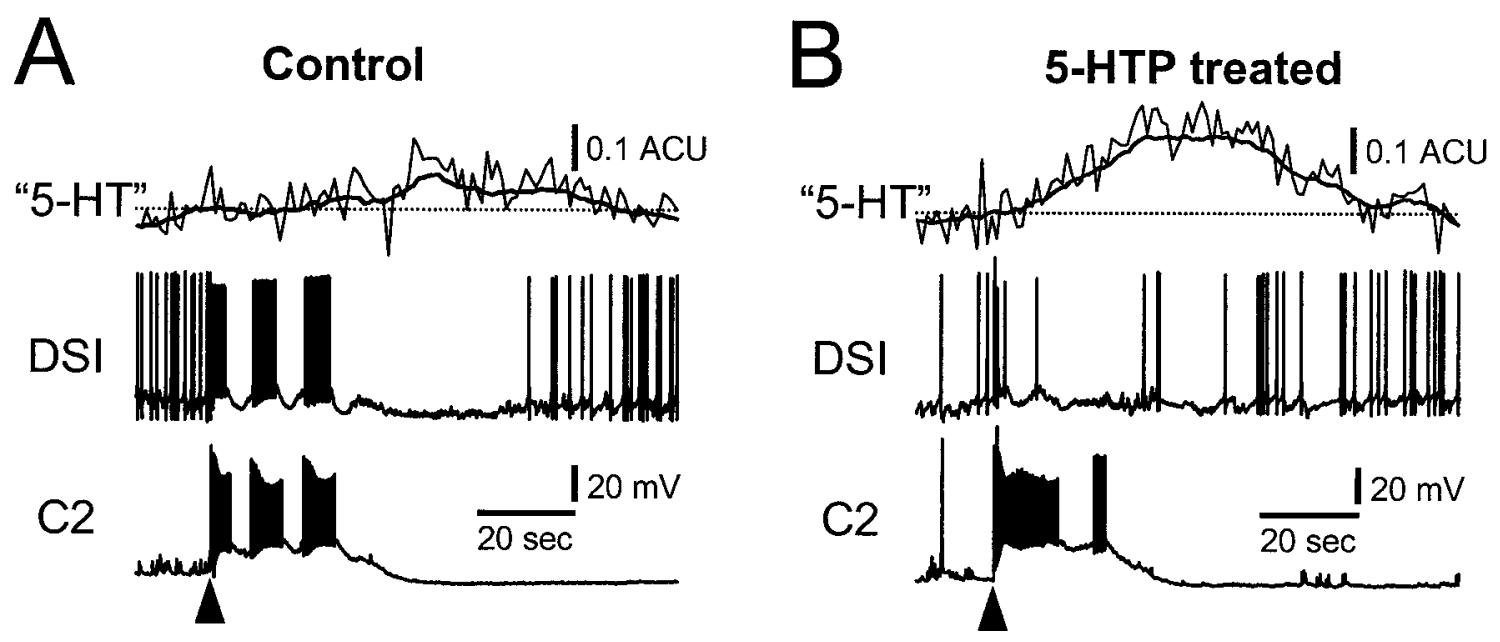

Figure 9. 5-HTP pretreatment increased the size of the electrochemical signal recorded in response to nerve stimulation. $A$. Before treatment, nerve stimulation (at arrowhead) evoked a small change in the signal compared to the prestimulus baseline (denoted by dotted line). The concomitant swim CPG output, recorded in a DSI and a C2, is also shown. B, The electrochemical signal recorded at the same site was greatly enhanced 3 hr after 5-HTP treatment $(2 \mathrm{~mm}, 30 \mathrm{~min}$ ). The DSI fired a brief burst of APs in response to nerve stimulation (at arrowhead) with minimal participation in the ensuing CPG activity, although C2 fired strongly. The electrochemical signal extends well beyond the end of depolarization in C2. The electrochemical traces (light trace) were digitally smoothed using a running average of 90 points to illustrate the slow changes (heavy trace). Recordings were obtained in normal saline.

requiring prolonged tonic spiking, a single burst of DSI spiking activity from a brief depolarization often evoked sustained rhythmic firing in $\mathrm{C} 2$ and other swim interneurons. During these bouts of activity, the DSIs were silent or minimally active (Fig. 6B). Thus, serotonin released from the DSIs may be sufficient to elicit rhythmic CPG activity.

\section{Increased 5-HT release may change CPG activity}

Over time, in 5-HTP-treated preparations, the DSIs became progressively less active in response to nerve stimulation, eventually losing their rhythmic bursting pattern and firing just a single burst. Although the DSIs ceased to fire in a bursting pattern, C2 continued to burst almost normally in many cases.

The nerve-evoked burst of DSI spikes in 5-HTP-treated preparations, although short-lived, may cause the prolonged spiking response of $\mathrm{C} 2$ and other neurons to nerve stimulation. As we showed, directly depolarizing a single DSI had a comparable effect on swim CPG activity. However, immunohistochemical data suggest that 5-HTP treatment increases the 5-HT levels of all serotonergic neurons. Thus, it is possible that spillover from serotonergic synapses not normally involved in the swim circuit contributes to the prolonged nerve-evoked response. Numerous serotonergic pedal neurons have axons in the nerve stimulated to elicit the swim motor program and may be activated by nerve stimulation. If spillover occurs, it indicates that participation in a neuronal circuit can be changed by altering transmitter synthesis, a novel concept for circuit organization.

The duration of CPG activation may reflect the time course of the 5-HT bolus released from serotonergic neurons, including the DSIs, or it may represent long-lasting actions of the 5-HT. The microvoltammetry recordings support the former mechanism because they suggest that extracellular 5-HT levels may be elevated for tens of seconds after nerve stimulation in 5-HTP-treated preparations.

\section{Feedback inhibition may underlie the loss of bursting} in the DSIs

The lack of ongoing DSI participation in the swim CPG output after 5-HTP treatment is likely attributable to enhanced negative feedback from two sources (Fig. 1A). First, the DSIs recruit inhibition onto themselves from a set of unidentified inhibitory interneurons (Getting et al., 1980; Getting and Dekin, 1985; Getting, 1989b). Enhanced DSI synaptic output may further recruit this inhibitory input, leading to increased DSI inhibition. Second, the connection from C2 to the DSIs is largely inhibitory (Getting, 1981). DSI-evoked neuromodulation increases the strength of this connection (Katz and Frost, 1994). Increasing 5-HT release from the DSIs should enhance C2 excitation and strengthen this inhibitory feedback connection.

Other serotonergic neurons exhibit functionally similar feedback inhibition, although the mechanisms vary. Serotonergic neurons in the lobster exhibit autoinhibition that is attributed to intrinsic properties of the cells and not actions of serotonin (Heinrich et al., 1999). In contrast, 5-HT release from dorsal raphe nucleus neurons is inhibited by 5-HT autoreceptors (Gothert, 1990; Sharp et al., 1997; Blier et al., 1998; Corradetti et al., 1998). We do not know if 5-HT autoreceptors limit 5-HT release from the DSI terminals. However, 5-HT directly excites isolated DSIs (Popova and Katz, 1998), and the DSIs monosynaptically excite each other in situ (Getting, 1981), suggesting that inhibition is produced by feedback from other cells.

\section{5-HT augmentation and central pattern generation}

Exogenously applied 5-HT and 5-HTP modulate GPG-generated rhythmic activity, including locomotion, swimming, respiration, and chewing, in many invertebrates and vertebrates (Willard, 1981; Hashemzadeh-Gargari and Friesen, 1989; Kabotyanski et al., 1990; Kabotyanskii and Sakharov, 1991; Satterlie and Norekian, 1995; Norekian and Satterlie, 1996). The effects of 5-HT on the leech swim-initiating interneuron, cell 204, is of special interest because 5-HT increases the ability of cell 204 to elicit swimming in a manner similar to the effect of 5-HTP treatment on DSI potency. Normally, cell 204 must be depolarized continuously to elicit sustained swimming, and the swimming ends soon after stimulation of cell 204 is stopped (Debski and Friesen, 1986). In 5-HT, brief depolarization of cell 204 is sufficient to elicit sustained swim episodes, attributable in part to 
changes in the electrical properties of cell 204 (Angstadt and Friesen, 1993a,b). It is possible that 5-HTP treatment also alters the membrane properties of DSI in Tritonia, possibly through long-term increases in extracellular 5-HT levels.

Serotonergic modulation also plays an important role in regulating swim activity in vertebrate species. Increased 5-HT levels reduce fictive swim burst frequency and increase burst intensity in the lamprey (Harris-Warrick and Cohen, 1985; Christenson et al., 1989), and 5-HTP treatment restores swimming activity in eels treated with para-chlorophenylalanine to reduce 5-HT levels (Genot et al., 1984). 5-HT and 5-HTP have modulatory effects on fictive swimming in Rana temporaria and Xenopus laevis, acting to increase burst duration and intensity while reducing cycle frequency and episode duration (Sillar et al., 1992; Woolston et al., 1994).

5-HTP has different effects on the Tritonia swim CPG than other systems heretofore described: 5-HTP treatment markedly alters the swim CPG output rather than evoking swims or altering burst duration or cycle frequency. This may be because 5-HT is an intrinsic neuromodulator in the Tritonia swim CPG. Because the serotonergic DSIs normally participate in cycle-by-cycle pattern generation by the CPG, it is likely that increasing the 5-HT content of the DSIs and other serotonergic neurons upsets the balance of the circuit itself and disrupts pattern generation.

\section{The effects of neurotransmitter supplementation on neuronal circuit operation}

The findings reported in this paper demonstrate the importance of circuit interactions for predicting the effects of drugs that alter transmitter content. As we have seen, increases in neurotransmitter release from a neuron might be counterbalanced by decreases in the activity of that neuron, possibly because of autoregulation. Furthermore, system-wide changes in transmitter levels may cause overflow from neighboring synapses and change the relative importance of particular neurons, suggesting that the effective makeup of a circuit can be artificially altered by increasing transmitter content.

\section{REFERENCES}

Abraham FD, Willows AOD (1971) Plasticity of a fixed action pattern in the sea slug Tritonia diomedia [sic]. Commun Behav Biol 6:271-280.

Angstadt JD, Friesen WO (1993a) Modulation of swimming behavior in the medicinal leech. I. Effects of serotonin on the electrical properties of swim-gating cell 204. J Comp Physiol [A] 172:223-234.

Angstadt JD, Friesen WO (1993b) Modulation of swimming behavior in the medicinal leech. II. Ionic conductances underlying serotonergic modulation of swim-gating cell 204. J Comp Physiol [A] 172:235-248.

Audesirk G (1985) Amine-containing neurons in the brain of Lymnaea stagnalis: distribution and effects of precursors. Comp Biochem Physiol 81A:359-365.

Blier P, Piñeyro G, El Mansari M, Bergeron R, De Montigny C (1998) Role of somatodendritic 5-HT autoreceptors in modulating 5-HT neurotransmission. Ann NY Acad Sci 861:204-216.

Boadle-Biber MC (1982) Biosynthesis of serotonin. In: Biology of serotonergic transmission (Osborne NN, ed), pp 63-94. Chichester: Wiley.

Byerley WF, Judd LL, Reimherr FW, Grosser BI (1987) 5-Hydroxytryptophan: a review of its antidepressant efficacy and adverse effects. J Clin Psychopharmacol 7:127-137.

Christenson J, Franck J, Grillner S (1989) Increase in endogenous 5-hydroxytryptamine levels modulates the central network underlying locomotion in the lamprey spinal cord. Neurosci Lett 100:188-192.

Cooper JR, Bloom FE, Roth RH (1996) The biochemical basis of neuropharmacology. New York: Oxford.

Corradetti R, Laaris N, Hanoun N, Laporte AM, Le Poul E, Hamon M, Lanfumey L (1998) Antagonist properties of (-)-pindolol and WAY 100635 at somatodendritic and postsynaptic 5-HT1A receptors in the rat brain. Br J Pharmacol 123:449-462.
Cottrell GA, Powell B (1971) Formation of serotonin by isolated serotonin-containing neurons and by isolated non-amine-containing neurons. J Neurochem 18:1695-1697.

Debski EA, Friesen WO (1986) Role of central interneurons in habituation of swimming activity in the medicinal leech. J Neurophysiol 55:977-994.

Dorsett DA, Willows AOD, Hoyle G (1969) Centrally generated nerve impulse sequences determining swimming behavior in Tritonia. Nature 224:711-712.

Frost WN, Katz PS (1996) Single neuron control over a complex motor program. Proc Natl Acad Sci USA 93:422-426.

Genot G, Conan GY, Barthelemy L, Peyraud C (1984) Effects of 5-HT serotonin on spontaneous locomotor activity of eels. Comp Biochem Physiol [C] 79:189-192.

Getting PA (1977) Neuronal organization of escape swimming in Tritonia. J Comp Physiol [A] 121:325-342.

Getting PA (1981) Mechanisms of pattern generation underlying swimming in Tritonia. I. Neuronal network formed by monosynaptic connections. J Neurophysiol 46:65-79.

Getting PA (1989a) A network oscillator underlying swimming in Tritonia. In: Neuronal and cellular oscillators (Jacklet JW, ed), pp 215-236. New York: Marcel Dekker.

Getting PA (1989b) Emerging principles governing the operation of neural networks. Annu Rev Neurosci 12:185-204.

Getting PA, Dekin MS (1985) Mechanisms of pattern generation underlying swimming in Tritonia. IV. Gating of central pattern generator. J Neurophysiol 53:466-480.

Getting PA, Lennard PR, Hume RI (1980) Central pattern generator mediating swimming in Tritonia. I. Identification and synaptic interactions. J Neurophysiol 44:151-164.

Gothert M (1990) Presynaptic serotonin receptors in the central nervous system. Ann NY Acad Sci 604:102-112.

Handley SL (1995) 5-hydroxytryptamine pathways in anxiety and its treatment. Pharmacol Ther 66:103-148.

Harris-Warrick RM, Cohen AH (1985) Serotonin modulates the central pattern generator for locomotion in the isolated lamprey spinal cord. J Exp Biol 116:27-46.

Hashemzadeh-Gargari H, Friesen WO (1989) Modulation of swimming activity in the medicinal leech by serotonin and octopamine. Comp Biochem Physiol [C] 94:295-302.

Heinrich R, Cromarty SI, Hörner M, Edwards DH, Kravitz EA (1999) Autoinhibition of serotonin cells: an intrinsic regulatory mechanism sensitive to the pattern of usage of the cells. Proc Natl Acad Sci USA 96:2473-2478.

Jacobs BL, Fornal CA (1997) Serotonin and motor activity. Curr Opin Neurobiol 7:820-825.

Kabotyanskii EA, Sakharov DA (1991) Neuronal correlates of the serotonin-dependent behavior of the pteropod mollusc Clione limacina. Neurosci Behav Physiol 21:422-435.

Kabotyanski EA, Milosevic I, Sakharov DA (1990) Neuronal correlates of 5-hydroxytryptophan-induced sustained swimming in Aplysia fasciata. Comp Biochem Physiol [C] 95:39-44.

Katz PS (1999) What are we talking about? Modes of neuronal communication. In: Beyond neurotransmission: the role of neuromodulation in information processing (Katz PS, ed), pp 1-28. New York: Oxford UP.

Katz PS, Frost WN (1995a) Intrinsic neuromodulation in the Tritonia swim CPG: the serotonergic dorsal swim interneurons act presynaptically to enhance transmitter release from interneuron C2. J Neurosci 15:6035-6045.

Katz PS, Frost WN (1995b) Intrinsic neuromodulation in the Tritonia swim CPG: Serotonin mediates both neuromodulation and neurotransmission by the dorsal swim interneurons. J Neurophysiol 74:2281-2294.

Katz PS, Frost WN (1997) Removal of spike frequency adaptation via neuromodulation intrinsic to the Tritonia escape swim central pattern generator. J Neurosci 17:7703-7713.

Katz PS, Kirk MD, Govind CK (1993) Facilitation and depression at different branches of the same motor axon: evidence for presynaptic differences in release. J Neurosci 13:3075-3089.

atz PS, Getting PA, Frost WN (1994) Dynamic neuromodulation of synaptic strength intrinsic to a central pattern generator circuit. Nature 367:729-731.

Launay JM, Callebert J, Bondoux D, Loric S, Maroteaux L (1994) Serotonin receptors and therapeutics. Cell Mol Biol 40:327-336.

Lennard PR, Getting PA, Hume RI (1980) Central pattern generator 
mediating swimming in Tritonia. II. Initiation, maintenance, and termination. J Neurophysiol 44:165-173.

Lookingland KJ, Shannon NJ, Chapin DS, Moore KE (1986) Exogenous tryptophan increases synthesis, storage, and intraneuronal metabolism of 5-hydroxytryptamine in the rat hypothalamus. J Neurochem 47:205-212.

McCaman MW, Ono JK, McCaman, RE (1984) 5-Hydroxytryptamine measurements in molluscan ganglia and neurons using a modified radioenzymatic assay. J Neurochem 43:91-99.

McClellan AD, Brown GD, Getting PA (1994) Modulation of swimming in Tritonia: excitatory and inhibitory effects of serotonin. J Comp Physiol [A] 174:257-266.

McLean IW, Nakane PK (1974) Periodate-lysine-paraformaldehyde fixative. A new fixation for immunoelectron microscopy. J Histochem Cytochem 22:1077-1083.

Norekian TP, Satterlie RA (1996) Cerebral serotonergic neurons reciprocally modulate swim and withdrawal neural networks in the mollusk Clione limacina. J Neurophysiol 75:538-546.

O'Neill RD (1994) Microvoltammetric techniques and sensors for monitoring neurochemical dynamics in vivo. Analyst 119:767-780.

Pentreath VW, Cottrell GA (1972) Selective uptake of 5-hydroxytryptamine by axonal processes in Helix pomatia. Nat New Biol 239:213-214.

Pentreath VW, Cottrell GA (1973) Uptake of serotonin, 5-hydroxytryptophan and tryptophan by giant serotonin-containing neurones and other neurones in the central nervous system of the snail (Helix pomatia). Z Zellforsch Mikrosk Anat 143:21-35.

Pothos E, Desmond M, Sulzer (1996) D L-3,4-dihydroxyphenylalanine increases the quantal size of exocytotic dopamine release in vitro. J Neurochem 66:629-636.

Popova LB, Katz PS (1998) Serotonin responses of acutely isolated identified neurons from the Tritonia swim CPG. Soc Neurosci Abstr 24:358.

Poulain B, Baux G, Tauc L (1986) Presynaptic transmitter content controls the number of quanta released at a neuro-neuronal cholinergic synapse. Proc Natl Acad Sci USA 83:170-173.

Satterlie RA, Norekian TP (1995) Serotonergic modulation of swim- ming speed in the pteropod mollusc Clione limacina. III. Cerebral neurons. J Exp Biol 198:917-930.

Schlapfer WT, Woodson PBJ, Tremblay JP, Barondes SH (1974) Depression and frequency facilitation at a synapse in Aplysia californica: Evidence for regulation by availability of transmitter. Brain Res 76:267-280.

Sharp T, Umbers V, Gartside SE (1997) Effect of a selective 5-HT reuptake inhibitor in combination with 5-HT1A and 5-HT1B receptor antagonists on extracellular 5-HT in rat frontal cortex in vivo. $\mathrm{Br} \mathrm{J}$ Pharmacol 121:941-946.

Sillar KT, Wedderburn JFS, Simmers AJ (1992) Modulation of swimming rhythmicity by 5 -hydroxytryptamine during post-embryonic development in Xenopus laevis. Proc R Soc Lond B Biol Sci 250:107-114.

Silverstone T (1992) Appetite suppressants. A review. Drugs 43:820-836.

Sternbach H (1991) The serotonin syndrome. Am J Psychiatry 148: $705-713$.

Sudlow LC, Jing J, Moroz LL, Gillette R (1998) Serotonin immunoreactivity in the central nervous system of the marine molluscs Pleurobrachaea californica and Tritonia diomedea. J Comp Neurol 395: $466-480$

Taghert PH, Willows AOD (1978) Control of a fixed action pattern by single, central neurons in the marine mollusk, Tritonia diomedea. J Comp Physiol 123:253-259.

Verhage M, Ghijsen WEJM, Lopes da Silva FH (1994) Presynaptic plasticity: the regulation of $\mathrm{Ca}^{2+}$-dependent transmitter release. Prog Neurobiol 42:539-574.

Willard AL (1981) Effects of serotonin on the generation of the motor program for swimming by the medicinal leech. J Neurosci 1:936-944.

Willows AOD, Hoyle G (1969) Neuronal network triggering of fixed action pattern. Science 166:1549-1551.

Woolston A-M, Wedderburn JFS, Sillar KT (1994) Descending serotonergic spinal projections and modulation of locomotor rhythmicity in Rana temporaria embryos. Proc R Soc Lond B Biol Sci 255:73-79.

Zucker RS (1989) Short-term synaptic plasticity. Annu Rev Neurosci 12:13-31. 